\title{
Epigenetics: development, dynamics and disease
}

\author{
Tanja Vogel • Silke Lassmann
}

Published online: 14 May 2014

(C) Springer-Verlag Berlin Heidelberg 2014

With the discovery of epigenetic mechanisms that regulate the development and function of cells and tissues, the life sciences have entered a novel area of disease-relevant research. Epigenetic modifications can be highly dynamic alterations in DNA and chromatin and, thereby, they differ from changes at the level of the DNA sequence itself, i.e. by mutations, or at the level of DNA structure, e.g. translocations. Epigenetic mechanisms are central to the regulation of cell-type-specific physiology and pathology. Cell-specific gene expression and hence cellular phenotypes are epigenetically controlled by marking histones through chemical modifications and DNA through methylation, but also through other mechanisms such as incorporation of histone variants, transcription of noncoding RNAs, RNA editing and chromatin remodelling. These epigenetic mechanisms are discussed in this special issue from various angles including basic, translational and applied clinical research. The fundamental understanding of epigenetic mechanisms opens a new window for changing the transcriptional states of cells, tissues and organs, both in physiological and disease states.

Tanja Vogel and Silke Lassmann are members of the DFG Collaborative Research Center 992 "Medical Epigenetics". Silke Lassmann is an associated member of BIOSS Centre for Biological Signalling Studies, University of Freiburg, Freiburg, Germany, and is also a member of the German Consortium for Translational Cancer Research (DKTK) and German Cancer Research Center (DKFZ), Heidelberg, Germany.

T. Vogel $(\bowtie)$

Department of Molecular Embryology, Institute for Anatomy and

Cell Biology, Albertstrasse 17, Freiburg 79104, Germany

e-mail: tanja.vogel@anat.uni-freiburg.de

S. Lassmann

Department of Pathology, Institute of Surgical Pathology, University

Medical Center, Breisacherstr. 115A, Freiburg 79106, Germany

e-mail: silke.lassmann@uniklinik-freiburg.de
With the selection of the articles and viewpoints within this special issue, our aim here is to foster cross talk between basic and applied epigenetic science. Such strong interdisciplinary research should lead to potential future benefits for improved medical care of patients that suffer from diseases in which epigenetic functions are derailed, or even for preventive measures for avoiding the development of epigenetically driven diseases. The following provides an overview of the topics discussed in this special issue of Cell and Tissue Research on "Epigenetics".

The series of articles starts with recent advances in basic research to improve our understanding of the roles of histone variants, the dynamics during cell division, signal transduction to chromatin, the view of specific regulatory elements in the genome, interaction between chromatin modifiers and non-coding RNAs, and the role of RNA editing. The article by Biterge and Schneider (2014) gives a comprehensive overview of histone variants and their peculiarity with regard to temporal expression patterns and mRNA characteristics. Incorporation of variants within the chromatin broadens the plethora of mechanisms for regulating gene expression, as these variants not only influence nucleosome stability and shape distinct chromatin domains, but are also implicated in DNA repair. Although many aspects are not fully understood as yet, e.g. the function of histone variants and their recruitment and incorporation into chromatin, growing evidence indicates that the variants are of clinical importance.

The role of histone modifications in chromatin dynamics in the cell cycle is presented by Doenecke (2014). Within this classical field of epigenetics, the author provides a comprehensive overview of the implications of histones in the transition from an open chromatin conformation in the interphase to a tightly packed chromatin that is the hallmark of mitotic chromosomes. Within this transition, histone modifications and histone variants play pivotal roles in the various accompanying processes. These include the establishment of 
replication-competent chromatin, replication itself, condensation of chromosomes, assembly of kinetochores and finally the reversal of these structural features after successful cell division. DNA methylation, histone modifications and histone variants in this context are epigenetic traits that serve to pass information through phases of structural perturbation to ensure persistent gene expression in the next cell generation.

Of special interest is an understanding how chromatin modifications and thereby the regulation of gene expression are directed to specific genomic regions. Scientific efforts are directed to identify the way that locus-specific recruitment of chromatin-modifying enzymes is achieved and the signalling pathways that are involved to coordinate cell extrinsic instructive cues and cell intrinsic interpretation of the former. The following three reviews in this issue summarise recent advances in resolving these open questions.

The contribution of Kolybaba and Classen (2014) elaborates on the signalling pathways that regulate Polycomb and Trithorax protein activity. Specifically, the authors discuss the way that signals triggered by kinase cascades, metabolic fluctuations and cell cycle dynamics influence the function of Polycomb and Trithorax. These protein complexes are important for development and stem cell differentiation and also under conditions of misexpression, namely cancer. In particular, this article suggests that cellular stress is an extrinsic signal that activates chromatin-modifying enzymes. Among the emerging signalling effectors, the authors discuss, for example, mitogen-activated protein kinase (MAPK), extracellular signal-regulated kinase (ERK), p38 and c-Jun NH2terminal kinase (JNK), phosphoinositol-3-kinase (PI3K), protein kinase $\mathrm{B}(\mathrm{PKB} \alpha / \beta / \gamma ; \mathrm{AKT})$, and mitogen-activated protein kinase-activated protein kinase 2 (MK2), all of which are implicated in Polycomb protein regulation. These data not only suggest that Polycomb function is highly dynamic and can be altered in response to environmental cues, but they also raise a question about the impact of signalling in epigenetic events in general. Furthermore, we need to understand whether the fast kinetics of signalling cascades can modify chromatin signature in such a way that it can be stably transmitted to the next cell generation, an important hallmark in the definition of epigenetics. Another possible view is that signalling to chromatin is a dynamic mode for regulating gene transcription without having an impact on the next generation.

Friedensohn and Sawarkar (2014) review recent findings about the significance of genetic variations in intergenic regions for personalised medicine. They embark on the finding that most disease-associated loci map to non-coding regions of the genome. These intergenic regions are now widely accepted to harbour important regulatory elements to drive or repress gene expression. Genetic variability in these socalled cis-regulatory regions (CRR) accounts for differential expression levels by influencing the flanking chromatin architecture. Furthermore, they have been implicated in post-transcriptional gene expression and the splicing and timing of DNA replication. The review highlights recent studies that unravel the relationship of transcription factors, chromatin and CRR variations and their implication in health and evolution. Identification of CRRs within the genome is an emerging field that profits from high throughput sequencing data of large populations. Family studies enrich these efforts and provide information about variability whenever a CRR is identified. CRR variations not only account for phenotypic differences, but also monitor evolutionary potential, as they are involved in natural selection and adaptation. Prediction of the outcome of a specific CRR variation might be developed as a powerful tool for personalised medicine, emphasising the potential of this aspect of epigenetic transcriptional regulation.

An emerging field in epigenetics is the analysis of the interdependencies of various modes of action. Among such cross talk are the Histone $\mathrm{H} 3$ methylation at lysine 27 (H3K27) that serves as a template for re-establishing DNA methylation during early development, and the Histone $\mathrm{H} 3$ methylation at lysine $4(\mathrm{H} 3 \mathrm{~K} 4)$ that recruits DNA demethylating activities such as Gadd45 proteins. The article by Backofen and Vogel (2014) focuses on another layer of complexity and highlights recent findings on the interaction of chromatin-modifying enzymes and long non-coding RNAs (lncRNAs) in transcriptional control. A comprehensive view of recent findings concerning interactions of IncRNAs and DNA methyltransferases, Polycomb and Trithorax proteins, histone acetyltransferases and deacetylases, histone methyltransferases and chromatin remodellers is accompanied by a summary of recent developments in bioinformatics. Bioinformatics is a necessary prerequisite to analyse high throughput data sets, to annotate lncRNAs and to provide comprehensive hypotheses for putative biological functions to "feed" wet-lab scientists. The article places emphasis on the identification of IncRNAs within the genome and the analyses of RNA-protein interaction datasets that are emerging through novel methods such as RIP-, CLIP-, CHIRPand CLASH-seq. The article demonstrates that close collaboration between bioinformatics and wet-lab scientists is needed for efficient and successful data generation, analyses and interpretation.

In pursuit of the action of RNA in the epigenetic control of gene expression and protein translation, the next set of articles describes the role of RNA editing as an epigenetic posttranscriptional process. Tomaselli, Locatelli and Gallo (2014) discuss the mechanisms of the A-to-I deaminase enzymes, ADARs, and their implication in human diseases, such as neurological and neurodegenerative diseases and also cancer. The article summarises the various cellular processes in which ADARs are involved. It provides a view of the way that these enzyme activities can be targeted to correct RNA editing dysfunction and whether they are useful tools for therapeutic 
approaches, e.g. to correct diseases not caused by RNA editing but through other mutations.

The review provided by Morrison (2014) speculates that RNA editing through ADARs is implicated in organ and tissue regeneration. This article focuses on a physiological setting that requires a cellular environment to foster regeneration, e.g. it has to provide a given structural stability and shape and a signalling compartment allowing cell renewal and differentiation. In the newt, limb regeneration accompanies elevated levels of ADAR expression and a high rate of A-to-I editing.

With regard to the physiology of chromatin modifications, this issue contains further views on the implication of epigenetic mechanisms for specific organ development und function, with emphasis on the central nervous system (CNS) and consideration of heart and kidney development.

The article by Roidl and Hacker (2014) gives a comprehensive overview about the methylation of histones and their role in central nervous development. Histone methylations are dynamically added and removed by a variety of enzymes. The authors' view spans from basic concepts of different histone methylations to their implication in neural stem cell biology. Here, the authors discuss the histone-mediated control of the neurogenic developmental program of embryonic stem cells, a standard approach to studying basic mechanisms of differentiation. Furthermore, the article provides insight into in vivo models that have been used to understand the function of histone methylation in nervous system development. This field is only just opening up and will provide important information about the specificity of action of the various enzymes that are involved in histone methylation. In this regard, learning whether enzymes with the same activity have redundant or specific functions and the way in which this is achieved will be of great interest.

Sheikh (2014) highlights gene activation through histone acetylation in the context of neural development. The orchestrated process of cell proliferation and differentiation during brain development is influenced through cell extrinsic and intrinsic signals that often converge on histone acetyltransferases (HAT) and their counterparts, histone deacetylases (HDAC). Both classes of chromatin-modifying enzymes are important interpreters of developmental signals and are necessary for proper brain function. The review highlights the functions of various HAT in the nervous system and provides an overview of the way that these molecules affect proliferation, migration, differentiation and survival of neural progenitors and mature cells. Improper functions of HAT are associated with CNS diseases. Thus, developing and mature cells control and coordinate the expression of HAT in a temporospatial manner. Given this important role for CNS development, the understanding of HAT activation, downstream signalling and effectors and their interaction with other epigenetic modifications is still unexplored and will provide an important field for further research.
Ramanathan and Tuoc (2014) examine another field of epigenetic mechanisms and share their view on the way that chromatin remodelling enzymes influence neural differentiation. They focus on the so-called BAF complexes, which are large protein assemblies with variable composition. BAF proteins confer chromatin dynamics through positioning the nucleosomes. Depending on the presence of specific protein components, these complexes have been implicated in the generation of distinct neural cell types and even trans-differentiation between cell types. The combinatorial assembly of the different BAF complex proteins mediates locus specificity and has various functions in the different stages of neural development. The authors further describe that BAF complexes interact with various transcription factors to confer their diverse influence on neural stem cell development.

BAF complexes are not only involved in neural stem cell development, but also in cardiac development and function, as outlined in the article by Nührenberg et al. (2014). The authors of this review highlight recent novel insights into the crucial roles of the chromatin remodelling by BAF complexes, DNA methylation and histone modifiers in cardiac development and disease. In particular they emphasise that altered epigenetic events contribute to fetal gene reactivation in cardiac hypertrophy and cardiac failure and to gene expression patterns, which are altered in diseases such as cardiomyopathy. Moreover, the article refers to inhibitors, which target, for example, bromodomain-containing proteins and are able to reverse specific pathological phenotypes, such as cardiac hypertrophy. Thus, this article nicely depicts the putative exploitation of epigenetic control mechanisms in the translation of basic research findings into a clinically relevant setting.

Furthermore, the power of epigenetics in translational research is pointed out by the article of Bechtel and Huber (2014), who focus on the epigenetic regulation of renal development, kidney disease occurrence and progression. Specifically, thehe authors discuss the epigenetic mechanisms that establish epithelial cell specification of nephrons and that are maintained to allow normal renal function. The authors highlight important regulators of DNA methylation, whose alteration might contribute to the loss of nephron numbers and the occurrence of proteinuria, hypertension and chronic kidney disease. In this context, the article refers to a recent clinical study (chronic renal insufficiency study) in which the altered $\mathrm{CpG}$ island methylation of genes involved in inflammatory and oxidative stress responses parallels the loss of kidney function. In addition, the authors discuss important experimental data concerning histone modifications in mouse kidney development. Here, they mention that a selective histone mark (H3K79me3) and histone methyltransferase (DOT1L) become elevated upon postnatal renal maturation, and guide the differentiation of renal epithelium into glomerular 
podocytes and into proximal and distal tubules. This implies that the disruption of this highly selective epigenetic modification causes severe developmental defects and results later in the loss of kidney homeostasis.

Thus, epigenetic mechanisms are widely associated with diseases if their proper function or control is disrupted, as discussed for the heart and kidney. Another set of articles in this CTR Special Issue therefore highlights the contribution of epigenetic modifiers to neurological diseases and selected cancers. The recent views in this important and growing field can only be partially addressed for selected cancers within this special issue of Cell and Tissue Research.

Staszewski and Prinz (2014) review current knowledge about epigenetic modifications within glial cells, which make up the greatest proportion of the CNS but are rarely studied with regard to transcriptional control through epigenetic mechanisms. Given the strong involvement of glial cells in neurodevelopment, neuroinflammation and neurodegeneration, this field is of particular interest for clinical therapies. In addition, specific epigentic regulation by histone modifications, such as acetylation, have been implicated in most pathological states of the nervous system. However, functional and mechanistic data are rarely found in the current literature. This review collects together the key questions relevant to this emerging field and hopefully will stimulate research in this direction.

With regard to the central concept of epithelial-to-mesenchymal transition (EMT) and its reverse, mesenchymal-toepithelial transition (MET), for cancer progression and metastasis, Mishra and Johnsen (2014) provide a comprehensive overview of the epigenetic and transcription factor plasticity associated with cellular changes during EMT/MET. Further, the authors specifically identify novel approaches of targeting cancer cells. They focus on the EMT/MET central regulator E-Cadherin (CDH1), whose expression is significantly altered by changes in DNA methylation within the $\mathrm{CpG}$ islands of the $\mathrm{CDH} 1$ promoter. This is, in part, guided by associated dimethylated Histone $\mathrm{H} 3$ at lysine 9 (H3K9me2). Similarly, the SUV39H1-mediated methylation of H3K9 results in DNA methylation and repression of the CDH1 promoter. This event is initiated via the interaction of the EMT-/ MET-associated transcription factor SNAIL with SUV39H1. This finding underlines the cross-talk of the epigenetic and transcription-factor-mediated regulation in EMT/MET. In addition, the authors comment on several epigenetic inhibitors, specifically targeting DNMTs and HDACs, and their potential to interfere with the EMT process.

The importance of DNA methylation in cancer cells is further specified in the article by Pfeifer et al (2014), who present key findings and perspectives on the role of a nonclassical type of DNA methylation, 5-methylcytosine $(5 \mathrm{mC})$ and its enzymatic oxidation product 5hydroxymethylcytosine $(5 \mathrm{hmC})$. Several experimental models and some cancer types suggest a close link between mutations in selected genes (TET and IDH family) and the epigenetic feature of $5 \mathrm{hmC}$. However, the majority of cancer types with $5 \mathrm{hmC}$ occurrence lack these mutations. Thus, Pfeifer and colleagues discuss current knowledge about the active oxidation-dependent pathway of $5 \mathrm{hmC}$ generation in diverse cancer types, the role of $5 \mathrm{hmC}$ as a biomarker and its association with classical DNA methylation $(5 \mathrm{mC})$. Moreover, the influence of $5 \mathrm{hmC}$ on TET and IDH and other epigenetic modifiers, such as EZH, MLL, DNMT, and viceversa, are depicted for key cancer entities.

In the final two articles of this CTR special issue, epigenetic regulation is viewed from the translational molecular pathology perspective in two epithelial cancer entities, i.e. carcinomas of the esophagus and of the breast.

Ahrens and co-authors (2014) focus on upper gastrointestinal tract cancers, namely esophageal squamous cell carcinomas and (Barrett's) adenocarcinomas. The authors critically discuss the current knowledge on epigenetic regulation at the level of micro RNAs (miRNA), DNA methylation and histone modifications observed in human tissue specimens. The article presents epigenetic alterations that have been identified in the development and progression of the two histotypes of esophageal cancer. Further, the authors provide information about epigenetic modifications as prognostic and/ or predictive markers for improved patient stratification. Finally, the review highlights epigenetic regulators that bear the potential to serve as therapeutic targets.

In a similar applied approach, Lehmann (2014) critically discusses the novel aspect of aberrant DNA methylation of miRNA genes in breast cancers and thereby touches on two hot topics in translational research. Importantly, the author provides insights into specific miRNAs and their regulation in the various subtypes of breast cancers and emphasizes their role in the EMT process of breast cancer cells. Moreover, Lehmann highlights a study showing a negative-feedback loop between DNMT1 and selected miRNAs in human tissue specimens of breast cancers and provides an outlook towards innovative fields, such as alterations of miRNAs by RNA methylation.

In summary, this collection of review articles aims to bring together knowledge of basic, translational and applied research in the field of epigenetics. We direct attention to several known mechanisms of physiological and pathological processes and by doing so we support the idea that the time for "medical epigenetics" is evolving in a fast pave.

\section{References}

Ahrens T, Werner M, Lassmann S (2014) Epigenetics in esophageal cancers. Cell Tissue Res. doi:10.1007/s00441-014-1876-y

Backofen R, Vogel T (2014) Biological and bioinformatical approaches to study crosstalk of long-non-coding RNAs and chromatin-modifying proteins. Cell Tissue Res. doi:10.1007/s00441-014-1885-X 
Bechtel W, Huber TB (2014) Chromatin dynamics in kidney development and function. Cell Tissue Res. doi:10.1007/s00441-014-1884-y

Biterge B, Schneider R (2014) Histone variants: key players of chromatin. Cell Tissue Res. doi:10.1007/s00441-014-1862-4

Doenecke D (2014) Chromatin dynamics from S-phase to mitosis: contributions of histone modifications. Cell Tissue Res. doi:10.1007/ s00441-014-1873-1

Friedensohn S, Sawarkar R (2014) Cis-regulatory variation: significance in biomedicine and evolution. Cell Tissue Res. doi:10.1007/s00441$014-1855-3$

Kolybaba A, Classen A-K (2014) Sensing cellular states — signaling to chromatin pathways targeting Polycomb and Trithorax group function. Cell Tissue Res. doi:10.1007/s00441-014-1824-x

Lehmann U (2014) Aberrant DNA methylation of microRNA genes in human breast cancer - a critical appraisal. Cell Tissue Res. doi:10. 1007/s00441-014-1793-0

Mishra VK, Johnsen SA (2014) Epigenomic regulation as a central regulator of the epithelialto mesenchymal transition during tumor progression. Cell Tissue Res. doi: 10.1007/s00441-014-1912-y

Morrison JI (2014) Editing our way to regeneration. Cell Tissue Res. doi: $10.1007 / \mathrm{s} 00441-014-1844-6$
Nührenberg T, Gilsbach R, Preissl S, Schnick T, Hein L (2014) Epigenetics in cardiac development, function and disease. Cell Tissue Res. doi:10.1007/s00441-014-1887-8

Pfeifer GP, Xiong W, Hahn MA, Jin S-G (2014) The role of 5hydroxymethylcytosine in human cancer. Cell Tissue Res. doi:10. 1007/s00441-014-1896-7

Ramanathan R, Tuoc TC (2014) Roles of chromatin remodeling BAF complex in neural differentiation and reprogramming. Cell Tissue Res. doi:10.1007/s00441-013-1791-7

Roidl D, Hacker C (2014) Histone methylation during neural development. Cell Tissue Res. doi:10.1007/s00441-014$1842-8$

Sheikh BN (2014) Crafting the brain - role of histone acetyltransferases in neural development and disease. Cell Tissue Res. doi:10.1007/ s00441-014-1835-7

Staszewski O, Prinz M (2014) Glial epigenetics in neuroinflammation and neurodegeneration. Cell Tissue Res. doi:10.1007/s00441-0141815-y

Tomaselli S, Locatelli F, Gallo A (2014) The RNA editing enzymes ADARs: mechanism of action and human disease. Cell Tissue Res. doi:10.1007/s00441-014-1863-3 